\title{
Research Paper: Wrong Belief of Emergency Delay: A Qualitative Content Analysis in Iranian Context
}

\author{
Elham Sepahvand $^{1,2}$ (D), Hamidreza Khankeh ${ }^{3,4^{*}}$ (D), Mohammadali Hosseini ${ }^{1}$, Behnam Akhbari ${ }^{5}$
}

1. Department of Nursing, University of Social Welfare and Rehabilitation Sciences, Tehran, Iran

2. Department of Nursing, Lorestan University of Medical Sciences, Khorramabad, Iran.

3. Research Center of Health in Emergency and Disasters, University of Social Welfare and Rehabilitation Sciences, Tehran, Iran.

4. Department of Clinical Science and Education, Karolinska Institutet, Södersjukhuset, Stockholm, Sweden.

5. Department of Physiotherapy, University of Social Welfare and Rehabilitation Sciences, Tehran, Iran

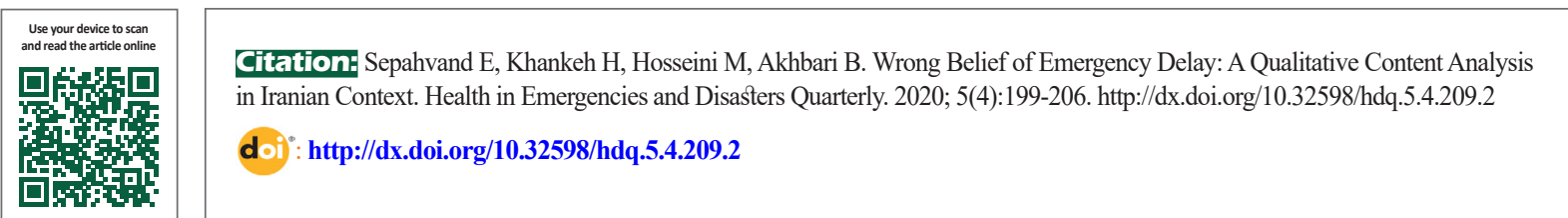

\section{(c) (i) (8)}

Article info:

Received: 17 Oct 2019

Accepted: 26 May 2020

Available Online: 01 Jul 2020

\section{Keywords:}

Qualitative study, Content analysis, Spinal cord injury, Road traffic injury

\section{ABSTRACT}

Background: During traffic accidents, professional staff provide care to the patient at the scene of the accident and then transport the victim to the hospital by ambulance. But sometimes this transition is carried out by relatives and laypeople. The purpose of this study was to explore the factors affecting people's involvement and transmission of the victims of the traffic accident.

Materials and Methods: This study was done with a qualitative content analysis method in 2018. The study participants were 16 people. In this study, a purposeful sampling method with maximum diversity was used. Semi-structured interviews were used to collect data using guiding questions. To observe the research ethics, the researcher, after obtaining permission from the University Ethics Committee, conducted interviews (IR.USWR.REC.1395.399).

Results: In total, 15 spinal cord injury victims and their relatives or laypeople and medical emergency technicians were interviewed. The class of wrong belief of delay was the main class in all interviews. Concepts such as past experiences, the pressure time, the lack of emergency time, cultural beliefs, and the tension explaining the wrong belief concept.

Conclusion: Wrong belief of delay was a concept that was extracted from the present study. It is recommended that the scene of the accident be examined in-depth and how to create a spinal cord injury in the injured with a grounded theory approach. 


\section{Introduction}

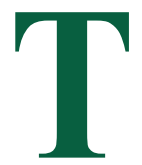

raumatic spinal cord injuries are a catastrophic global accident that often leads to permanent disability. Patients are suffering from spinal cord traumatic injuries emotionally and physically, and they also have functional problems after injury. It is imperative to identify patients with a high probability of having poor outcomes [1]. Early management of patients with potential spinal cord injury starts at the accident scene. The main concern during the early hours of the accident is that the neurological function of the injured might be impaired due to the pathological displacement of the damaged vertebrae. It is difficult to identify the signs of traumatic spinal cord injury in the acute phase, and emergency medical, and prehospital staff need to be trained about the early detection of signs and symptoms of spinal cord injury [2].

Pre-hospital care is a different and misunderstood concept in nursing and is sometimes defined as care that starts from the scene of the accident and ends in the hospital [3]. Emergency Medical Personnel (EMS) are responsible for providing pre-hospital care in many countries and serve as a link between pre-hospital care and inpatient care. One of the essential roles of prehospital services is to save lives and prevent further disabilities in the post-accident phase [4]. A small number of the injured receive care and treatment at the scene of the accident and are even transferred to the hospital by ambulance safely and appropriately. This transmission is mainly carried out by the relatives, untrained laypeople, and drivers of trucks [5].

At the scene of a traffic accident and when the injured is in a critical situation along with several relatives and friends, witnesses of the scene trying to help and save the lives of the injured and those affected by the accident [6]. In the study by Haghparast et al. (2010), factors such as the involvement of untrained people in the accident scene were significant obstacles for the provision of prehospital care [5]. Sometimes intervention of laypeople in the scene occurs following their ideas and beliefs for help. In the systematic review by Heydari et al. (2018), the performance of laypeople at the scene of traffic accidents was different in each religion and each country with different political and cultural values [7]. Cultural beliefs play an essential role in people's performance and their willingness to help. In the culture of Iran and many countries, a sense of altruism and helping one's fellow human beings provoke the person to help.
In many cases, this assistance has worsened the damage to the injured, and the result will be nothing but spinal cord injuries. Thus, it seems necessary to examine these behaviors and beliefs in the specific context of Iranian culture. Despite the importance of pre-hospital services in preventing mortality and disabilities following traffic accidents, there is insufficient knowledge, especially on cultural and contextual aspects [8]. Therefore, researchers aimed to conduct a study with a qualitative approach and content analysis method to discover the factors affecting the involvement and transmission of traffic accident casualties by ordinary people.

\section{Materials and Methods}

The current study was conducted by qualitative content analysis in 2018. Content analysis is a valid research method for data analysis. The study participants included 16 people, 7 of whom suffered spinal cord injuries with the experience of injury, 4 bystanders, and their entourage, as well as 5 emergency medical personnel. The research environment was Rafideh Rehabilitation Hospital and Shahid Jalaeipour Spinal Cord Injury Center in Tehran, and research participants were selected from these centers. The presence of the researcher as a nurse and clinical instructor, familiarity with the area, and access to knowledgeable and experienced people have been the reasons for choosing these centers. In this study, the purposeful sampling method was used. To consider the maximum variation in the subjects, an attempt was made to sample the spinal cord injury victims with different experiences.

Sampling was performed for 10 months until data saturation. Semi-structured interviews were used to collect data using guiding questions. The researcher first explained the purpose of the research to the participants, and then, the researcher interviewed them after obtaining written informed consent. Interviews were conducted using a tape recorder. Data saturation in the present study was reached from the $14^{\text {th }}$ interview. The participants were asked questions, such as "Please describe the scene of the accident from the beginning to the time of transmission to the hospital." Each interview also included follow-up questions such as "Can you explain more about this?" and "What did you mean by saying that?" In the end, the participants were asked to say what was left. The next questions were designed based on the participants' answers and the interview process.

Since the present study was a part of a more extensive grounded theory study with the approach of Strauss and Corbin (2015) (grounded theory), [9] constant data analysis method was used to analyze the data. Each interview 
was read and reviewed several times, and data analysis was performed using the inductive approach after gaining a general sense (i.e. without the researcher's preconceived notions). Important paragraphs were specified by reading line by line and underlining the necessary parts to distinguish them from other parts. Then the basic codes or the concept of levels one, two, and three were created. Finally, the content hidden in the data was introduced under the title of "theme" by comparing subclasses and classes with each other and deep and accurate reflection. Guba and Lincoln reliability criteria were used to achieve the accuracy and reliability of the data [10].

For data trustworthiness, the researcher took the following measures: 1. Long-term contact with the participants of different groups (triangulation) and gaining their trust; 2 . Using the interview guide; 3 . Allocating enough time to conduct interviews; 4. Constant comparison analysis of the data and classes in terms of similarities and differences; 5. Reviewing findings with participants; and 6. providing detailed data analysis and in-depth and rich research descriptions for readers. To observe the principles of research ethics, the researcher conducted the interviews after obtaining permission from the University Ethics Committee (IR.USWR.REC.1395.399).

\section{Results}

A total of 16 people with spinal cord injuries, their companions, witnesses, and emergency medical technicians were interviewed. Demographic information of the participants is given in Table 1 .

The "wrong belief in delay" was the main class seen in all the interviews. The belief of the injured, people on the accident scene, bystanders, and even rescue workers, such as the traffic police, on the delay of the emergency, was one of the main reasons for the transfer of the injured before the arrival of the rescue team. Concepts such as previous experiences of others, time pressure, lack of timely presence of the emergency, cultural beliefs, and stress explain the more abstract concept of misconceptions about the delay (Table 2), which are described separately.

\section{Previous experiences of the entourage}

The injured person is restless, asking for help, and having similar experiences of the injured person dying in accidents. All will create this mentality for the patient's companion and those around him that there is a possibility of death at any moment. Therefore, the injured should be transferred to the hospital as soon as possible. An example is provided below:

H4: "I was very anxious, I felt terrible, and I remembered the whole scene of my uncle's accident happened before led to his death. I was very stressed and anxious. I called the emergency every minute and waited for someone to come and help and do something."

Experiencing the death of loved ones in accidents increases the stress and accompanying anxiety. It makes the person consider the transfer of the patient as the first and only priority. In the meantime, the late arrival of the emergency reinforces the belief.

P1: "Yes, in one case, the car rolled over. The injured person inside the car was bleeding and had fractures. My car could not move. With the help of other people, we brought him out and took a passing car. We laid him nicely in the back seat and told the driver to get him to the hospital quickly. Every time we call the emergency, either the ambulance is late, or we are told that we only have one car and we cannot send more than one car due to another accident in another scene."

According to the extracted codes, this experience was not limited to companions, and the traffic police also experienced emergency department delays and for this reason, considers themselves responsible for transfer in the absence of the emergency.

\section{Time pressure}

It indicates that the companions and entourage will be in a temporary state of imbalance and time and situation unawareness after the accident. In this case, the decision is mainly based on emotion, and its logical and rational burden is reduced. Fear of the patient's death and loss of opportunity, as well as confusion and inability to distinguish between circumstances and situations, are among the cases which the patient's companion experiences at the scene of the accident. As the wife of the injured at the scene of the accident, one of the companions describes her experience:

H1: "I did not know where we were and what happened, I felt thunderstruck for a moment, and I went this way or that hitting my head. When I saw him in that situation, I felt worse, and he cried continuously and told me that his legs were not there. I was confused and did not know what to do".

Being in this situation makes the companion feel that every minute is lasting an hour or more. For this reason, 
Table 1. Demographic information of the study participants

\begin{tabular}{|c|c|c|c|c|c|c|}
\hline Row & Participant & Age (y) & Gender & Type of Experience & Interview Duration & Place of Interview \\
\hline 1 & $\mathrm{C} 1$ & 32 & Male & Injured & 35 & Rehabilitation Hospital \\
\hline 2 & C2 & 18 & Male & Injured & 30 & Rehabilitation Hospital \\
\hline 3 & C3 & 50 & Male & Injured & 35 & Rehabilitation Hospital \\
\hline 4 & C4 & 22 & Male & Injured & 30 & Rehabilitation Hospital \\
\hline 5 & C5 & 22 & Female & Injured & 20 & Molavi Rehabilitation Center \\
\hline 6 & C6 & 36 & Female & Injured & 40 & Molavi Rehabilitation Center \\
\hline 7 & $\mathrm{C7}$ & 19 & Female & Injured & 30 & Molavi Rehabilitation Center \\
\hline 8 & $\mathrm{~T} 1$ & 36 & Male & Technician & 35 & Rehabilitation Hospital \\
\hline 9 & $\mathrm{~T} 2$ & 35 & Male & Technician & 40 & University of Social Welfare Sciences \\
\hline 10 & T3 & 37 & Male & Technician & 40 & University of Social Welfare Sciences \\
\hline 11 & $\mathrm{~T} 4$ & 31 & Male & Technician & 30 & Private house \\
\hline 12 & T5 & 25 & Male & Technician & 25 & Home \\
\hline 13 & $\mathrm{H} 1$ & 28 & Male & Companion (spouse) & 30 & Molavi Rehabilitation Center \\
\hline 14 & $\mathrm{H} 2$ & 50 & Female & Companion (mother) & 25 & Molavi Rehabilitation Center \\
\hline 15 & H3 & 28 & Female & Companion (spouse) & 26 & Rehabilitation Hospital \\
\hline 16 & $\mathrm{H} 4$ & 26 & Male & Companion (child) & 30 & Molavi Rehabilitation Center \\
\hline
\end{tabular}

the patient companion feels that a long time has passed since the emergency was called and the rescue forces arrived, and they were late, and for this reason, he/she transfers the injured to the hospital. One of the emergency technicians says in this regard:

T2: "It is the belief of all people who have an accident that the emergency arrives late, while it is not true. Seconds are important to someone who has an accident or someone whose loved one is injured. Moreover, when a person is under much stress, the time lasts too long. The emergency may even arrive on time within 7 or 10 minutes, but they may say 'sir, you arrived an hour late'. While later, like many people, they go and complain about why the emergency arrived late. However, when timers are checked, they see that the emergency arrived exactly within 7 minutes".

\section{Lack of timely presence}

The next component extracted from the interviews is the lack of timely presence. Pre-hospital emergency personnel was not present in time at some of the acci- dent scenes, and the injured were taken to the hospital by the people before they arrived. Some of the reasons that prevented the timely arrival of the emergency to the accident scene were the multiple accidents that occurred in the area covered by an emergency base, the congestion of the accident scene, the traffic due to private cars congestion, and the impassability of the route. Examples of quotes in this area are provided as follows:

A participant arrived at the scene of the accident and was a witness of the accident, says:

L1: "We were waiting for the ambulance. It was late. Then we found out that the ambulance arrived after a few minutes, but it was so crowded, and the traffic was so heavy that it could not pass. So it stopped and waited for the road to be less crowded."

Police also talks about their experience at the scene of the accident: 
Table 2. Primary codes, classes, and subclasses of the "emergency delay belief" concept

\begin{tabular}{|c|c|}
\hline Subclasses & Primary Codes \\
\hline \multirow{5}{*}{ Time pressure } & Fear of the injured death \\
\hline & Confusion \\
\hline & Procrastination in emergency contact \\
\hline & Lack of situation awareness \\
\hline & Lack of time awareness \\
\hline \multirow{3}{*}{ Previous experiences of companions } & Previous experience of non-presence of the emergency team \\
\hline & Experience the death of loved ones in accidents \\
\hline & Experiencing similar scenes \\
\hline \multirow{5}{*}{ Lack of timely presence } & Obligation to comply with relief laws \\
\hline & The occurrence of accidents in a field \\
\hline & \\
\hline & Difficulty in finding the scene \\
\hline & The route is difficult to cross \\
\hline \multirow{10}{*}{ Cultural beliefs } & A sense of altruism \\
\hline & Eagerness to help \\
\hline & Witnessing the suffering of the injured \\
\hline & The injured disturbance \\
\hline & Emergency rescue need of the injured \\
\hline & Deterioration of the injured health status \\
\hline & \\
\hline & Inability to manage emotions \\
\hline & Entourage stress \\
\hline & Relief excitement \\
\hline
\end{tabular}

P1: "Every time we call the emergency, either ambulance is late, or they say we only have one car, and we could not send more than one car due to another accident".

In some cases, stress and anxiety of the scene and the existing psychological pressure have made the entourage not to call the emergency services. Therefore, it led to a lack of timely presence of the emergency to transfer the injured to the hospital. One of the injured companions at the scene says:

H2: "I felt so bad myself that I did not think about anything at all. I was so shocked that I forgot to call the emergency, but it seems that other people called later, but again, the emergency was late".

This ill-suited sense of responsibility on the part of the scene witnesses and the traffic police, which have a clear job description, is the result of believing in the emergency department delay. Moreover, in some cases, even the emergency services are not called. The following words confirm this:

P2: "We did not call the emergency at all because we could have taken them to the hospital ourselves. Of course, there are times when, for example, the injured person has a neck injury, and something worse may hap- 
pen to him, or, he might die God forbid. In such cases, we wait for the emergency to arrive. However, when we see that the injury is nothing special, we follow up and do it ourselves".

\section{Cultural beliefs}

Culture is a set of customs, beliefs, traditions, and social institutions that the people living within that culture consider themselves obliged to observe. In our country, altruism and helping each other are among the pervasive beliefs. At the scene of a traffic accident and when the injured is in a critical situation with several relatives and friends, witnesses try to help and save the lives of the injured and those affected by the accident. In the meantime, many actions inadvertently cause harm to the injured and leave irreparable consequences. One of the injured at the scene of the accident talks about his experience in this regard:

C10: "At the accident scene, people loved to help so much, and many of them caused harm to the injured. We are Iranians, and when we see an accident happens to somebody, we all mobilize and go for help, but we do not know how".

One of the technicians talks about people's beliefs in helping traffic accidents:

T5: "I think public aids are both good and bad, provided they are educated and cultured. Moreover, the person should get acquainted with the duties of the emergency and do not to think that the emergency only must take the patient to the hospital, they need to know that taking the patient correctly to the hospital is much more important. In any case, the sense of altruism and empathy they have may make them intervene. Our culture is such that when we see someone in trouble, we cannot ignore him".

\section{Tension}

Among other factors that can be seen at the accident scene is the tension among all people who are in close contact with the accident. This tension can affect participants in various dimensions, such as facing heartbreaking scenes, fear of facing the unknown, fear of facing with excited people, and the uncertainty of the condition of the injured. This tension between the people present at the scene and the patient's companions make them consider the arrival time of the rescue team more than what is real. Moreover, this makes them transfer the injured themselves. In this regard, a participant said:
T4: "Sometimes some scenes of accident hurt a lot For example, a mother dies in front of her children or a mother, while hitting her head, witnesses her child in a rolled-over car, and no one can help her".

The tension is increased when some people stop to see the incident on the side of the road. In some cases, this has caused the service provider to be unaware of the situation at the scene. One of the emergency technicians said:

T1: "There are stress and anxiety from the time of the call until arriving at the traffic accident area because the scene of traffic accidents is full of unknowns".

\section{Discussion}

This study aimed to investigate the effective factors in the involvement of people and the transmission of injured in traffic accidents. The results showed that during public participation, the witnesses of the accident scene tried to transfer the injured to the hospital before the arrival of the pre-hospital emergency team. They mentioned the belief in emergency department delay as the reason. Barriers such as traffic congestion by private cars near the accident scene or the impassability of the route will have negative consequences. This delay not only led to the late transmission of the injured to the hospital but also intensified the excitement of the entourage. It also led them to the belief that the emergency would always arrive late.

In this regard, in the study by Ali Nia et al., on explaining the barriers of pre-hospital services in traffic accidents, cases such as traffic congestion and the impassability of urban roads were the factors leading to the late arrival of the pre-hospital emergency on time at the accident scene [8]. Pourshaikhian in his grounded theory study stated that the delay in response time occurs due to traffic congestion, the impossibility of using GPS, difficulty in finding addresses and communication systems, all leading to restlessness and anxiety for the entourage, violence against emergency personnel, or transmission by companions before the arrival of the emergency personnel [11]. In the study by Haghparast, Khorasani, and Bigdeli, the traffic factor was also mentioned as an obstacle to achieving the desired relief services $[5,12,13]$.

Ali Nia et al. also stated in their study that sometimes pre-hospital emergency personnel has to stop the ambulance away from the scene and walk a distance. This way, not only service delivery is delayed, but leads, in some cases, to make mistakes in estimating the mission time, because the time interval between receiving a call and reaching the accident site is not calculated in 
the timing [6]. Previous experience of companions and scene witnesses regarding this late arrival can strengthen their belief and consequently intervene at the time of the next incident before the arrival of rescue personnel and transfer the injured to the hospital.

Another factor was the cultural beliefs. In the Iranian and many other cultures, a sense of altruism makes one to help. At the scene of a traffic accident and when the injured is in a critical situation along with several relatives and friends, witnesses of the scene trying to help and save the lives of the injured and those affected by the accident. In the meantime, many actions inadvertently cause harm to the injured and leave irreparable consequences. In the systematic review by Heydari et al., the performance of laypeople at the scene of traffic accidents was different in each religion and each country with different political and cultural values. Cultural beliefs play an essential role in people's performance and their willingness to help [7].

The results of the study by Haghparast et al. showed that the presence of people at the scene of the accident led to secondary injuries to the injured. They mentioned that factors such as a sense of altruism, excitement, curiosity, and a desire to help make the individuals consider themselves responsible for providing assistance and saving the patient's life. Unfortunately, this intervention at the scene of the accident had led to irreparable consequences [5]. However, the belief in helping and a sense of altruism can have positive results, if accompanied by sufficient knowledge and training in the field of first aid and, simultaneously, proper management at the accident scene.

Tensions among all those closely associated with the incident could also reinforce the belief that rescue workers arrived late. This tension is aroused in various dimensions, such as dealing with heartbreaking scenes, the unknown, excited people, and the uncertainty of the injured condition and can affect the participants. This tension among the relief forces causes them to leave the scene and lack of proper care. Moreover, the tension among the witnesses of the scene leads to disrupting the work of the rescue team and performing unwanted actions. This finding was also confirmed in the study by Ali Nia et al. [8].

The gathering of witnesses to see the incident on the side of the road can also increase the tension. In some cases, this has caused the service provider to be unaware of the situation at the traffic scene. Therefore, with proper management of the scene by relief teams, including the traffic police and law enforcement police, it is possible to minimize the crowd at the scene and prevent possible conflicts.

\section{Conclusion}

Misconception about rescue team delay was a concept extracted from the present study. The tension aroused at the scene, the companions' previous experiences of similar accidents, the time pressure, and the untimely presence of rescue workers reinforce this belief in the individuals. Thus, companions prefer to provide first aid themselves and transfer the injured to a medical center. The result of this misplaced and unprincipled transfer can be a secondary injury to the injured or aggravation of the initial injury. To investigate more deeply the accident scene and how the spinal cord injury is created in the injured, it is recommended that grounded-theory studies be conducted.

\section{Ethical Considerations}

\section{Compliance with ethical guidelines}

To observe the principles of research ethics, the researcher conducted the interviews after obtaining permission from the University Ethics Committee (IR. USWR.REC.1395.399).

\section{Funding}

The current article is extracted from the first author doctoral dissertation, supported by University of Social Welfare and Rehabilitation Sciences.

\section{Authors' contributions}

Conceptualization: First author and second author.; Methodology: second and third authors; Formal Analysis: First author, second author and third authors; Investigation: forth author; Writing-Original Draft Preparation all authors.

\section{Conflict of interest}

The authors confirm that there are no conflicts of interest.

\section{Acknowledgments}

The researchers would like to thank all the participants and officials of the Shahid Jalaeipour Spinal Cord Injury Center and Rafideh Kamal Rehabilitation Hospital. 


\section{References}

[1] Akmal M, Trivedi R, Sutcliffe J. Functional outcome in trauma patients with spinal injury. Spine. 2003; 28(2):180-5. [DOI:10.1097/00007632-200301150-00016] [PMID]

[2] Fransen BL, Hosman A, van Middendorp J, Edwards M, van Grunsven P, van de Meent H. Pre-hospital and acute management of traumatic spinal cord injury in the Netherlands: Survey results urge the need for standardisation. Spinal Cord. 2016; 54(1):34-8. [DOI:10.1038/sc.2015.111] [PMID]

[3] Froutan R, Khankeh HR, Mohammadi F. Concept analysis of pre-hospital care: A hybrid model. J Res Health. 2013; 3(3):412-21.

[4] Froutan R, Khankeh HR, Fallahi M, Ahmadi F, Norouzi K. Pre-hospital burn mission as a unique experience: A qualitative study. Burns. 2014; 40(8):1805-12. [DOI:10.1016/j. burns.2014.04.010] [PMID]

[5] Haghparast-Bidgoli $\mathrm{H}$, Hasselberg M, Khankeh H, Khorasani-Zavareh D, Johansson E. Barriers and facilitators to provide effective pre-hospital trauma care for road traffic injury victims in Iran: A grounded theory approach. BMC Emergency Medicine. 2010; 10:20. [DOI:10.1186/1471227X-10-20] [PMID] [PMCID]

[6] Alinia S. Exploring the process of prehospital care service in traffic injuries in Tehran. Tehran: University of social welfare and rehabilitation sciences; 2015.

[7] Heidari M, Aryankhesal A, Khorasani-Zavareh D. Laypeople roles at road traffic crash scenes: A systematic review. Int J Inj Contr Saf Promot. 2019; 26(1):82-91. [DOI:10.1080/174573 00.2018.1481869] [PMID]

[8] Alinia S, Khankeh H, Maddah SSB, Negarandeh R. Barriers of pre-hospital services in road traffic injuries in Tehran: The viewpoint of service providers. Int J Community Based Nurs Midwifery. 2015; 3(4):272-82. [PMID] [PMCID]

[9] Corbin J, Strauss A. Basics of qualitative research. New York: Sage; 2014. https://us.sagepub.com/en-us/nam/basics-ofqualitative-research/book 235578

[10] Schwandt TA, Lincoln YS, Guba EG. Judging interpretations: But is it rigorous? Trustworthiness and authenticity in naturalistic evaluation. New Dir Eval. 2007; 2007(114):11-25. [DOI:10.1002/ev.223]

[11] Pourshaikhian M, Khorasani-Zavareh D, Gorji H, Aryankhesal A, Barati A. Workplace violence process against emergency medical services staffs: A grounded theory. Glob J Health Sci. 2016; 8(12):213-24. [DOI:10.5539/gjhs.v8n12p213]

[12] Khorasani-Zavareh D, Khankeh HR, Mohammadi R, Laflamme L, Bikmoradi A, Haglund BJA. Post-crash management of road traffic injury victims in Iran. Stakeholders' views on current barriers and potential facilitators. BMC Emerg Med. 2009; 9(1):8. [DOI:10.1186/1471-227X-9-8] [PMID] [PMCID]

[13] Bigdeli M, Khorasani-Zavareh D, Mohammadi R. Pre-hospital care time intervals among victims of road traffic injuries in Iran. A cross-sectional study. BMC Public Health. 2010; 10(1):406. [DOI:10.1186/1471-2458-10-406] [PMID] [PMCID] 